\title{
Viscous Potential Flow Analysis of Electroaerodynamic Instability of a Liquid Sheet Sprayed with an Air Stream
}

\author{
Mukesh Kumar Awasthi, ${ }^{1}$ Vineet K. Srivastava, ${ }^{2}$ and M. Tamsir ${ }^{3}$ \\ ${ }^{1}$ Department of Mathematics, University of Petroleum and Energy Studies, Dehradun 248007, India \\ ${ }^{2}$ ISRO Telemetry, Tracking and Command Network (ISTRAC), Bangalore 560058, India \\ ${ }^{3}$ Department of Mathematics, Graphic Era University, Dehradun 248002, India
}

Correspondence should be addressed to Mukesh Kumar Awasthi; mukeshiitr.kumar@gmail.com

Received 19 May 2013; Accepted 31 July 2013

Academic Editor: Abdelali El Aroudi

Copyright (C) 2013 Mukesh Kumar Awasthi et al. This is an open access article distributed under the Creative Commons Attribution License, which permits unrestricted use, distribution, and reproduction in any medium, provided the original work is properly cited.

\begin{abstract}
The instability of a thin sheet of viscous and dielectric liquid moving in the same direction as an air stream in the presence of a uniform horizontal electric field has been carried out using viscous potential flow theory. It is observed that aerodynamic-enhanced instability occurs if the Weber number is much less than a critical value related to the ratio of the air and liquid stream velocities, viscosity ratio of two fluids, the electric field, and the dielectric constant values. Liquid viscosity has stabilizing effect in the stability analysis, while air viscosity has destabilizing effect.
\end{abstract}

\section{Introduction}

The past decade has witnessed a rapid advancement in the study of instability of a liquid sheet because of its importance in several scientific and technological processes. Application of plane liquid sheets is an interesting phenomenon, which can be seen in power generation and propulsion systems [1], chemical and pharmaceutical processes [2], surface curtain coatings, and in the adhesive industry [3]. A host of efforts have been devoted to study the behavior of a thin liquid sheet sprayed with an air stream. The instability and breakup process of a thin inviscid liquid sheet in a stationary gaseous medium have been investigated by Squire [4] and Hagerty and Shea [5]. Their results show that the surface tension resists the development of instability in a liquid sheet.

Fraser [6] has defined four modes of disintegration of a liquid sheet, namely, rim, wavy sheet, perforated sheet, and air impact. In rim, the disintegration takes place due to the contraction of the liquid sheet edges under the effect of surface tension. In wavy sheet, the disintegration occurs due to any small protuberance on the sheet which is subjected to two opposing forces: surface tension force, which draws the liquid back to the original undisturbed shape, and aerodynamic force, which pulls the liquid outward.
If the aerodynamic force exceeds to the surface tension force, then any small disturbance present in the sheet will grow rapidly, causing sheet instability. In perforated sheet disintegration, disturbances on the sheet puncture it when the sheet becomes thin enough, and the resulting holes expand regularly by surface tension until they coalesce, forming threads. In air impact disintegration, the disruption of the liquid is very near to that of a twin-fluid nozzle, where two streams of air and liquid are caused to impinge together. Squire [4] has studied the disintegration according to the wavy sheet mode. Dombrowski and Johns [7] extended the above analysis including the effect of liquid viscosity, and their results are valid for very large Weber number. Joostan et al. [8] have studied the effect of capillary waves on a free and viscous liquid sheet. Rashed et al. [9] have studied the disintegration of the liquid sheet according to perforated sheet mode and found that instability occurs if the Weber number is less than a critical value related to the ratio of the air and liquid stream velocities.

Ibrahim and Jackson [10] have studied the spatial instability of an incompressible liquid sheet injected into a compressible gas and found that the growth rate of disturbances increases initially with gas compressibility, reaches a maximum near gas Mach number 1, and then decreases 
as the gas Mach number is increased further. Ibrahim and Akpan [11] have studied the linear stability of inviscid liquid sheet emanated into an inviscid gas medium. A power series mathematical solution for the problem of instability of an inviscid liquid sheet of parabolic velocity profile emanated from a nozzle into an inviscid gas has been established by Ibrahim [12]. Nonlinear instability and breakup of an annular liquid sheet have been modeled by Ibrahim and Jog [13].

There is increasing interest in the electrohydrodynamic stability as the electric field plays an important role in many practical problems of biophysics, chemical engineering, and various other fields. The effect of an applied electric field on a liquid sheet has several applications in astrophysics, chemical engineering, and industry [14]. If an electric field is applied on a liquid sheet, the instability conditions will be considerably modified. El-Sayed [15] has studied the instability of a thin sheet of dielectric liquid moving in the same direction as an air stream in the presence of a uniform horizontal electric field and observed that the electric field has stabilizing effect, and there exists a critical Weber number above which instability is suppressed by the surface tension effect.

Viscous potential flow theory [16] has played an important role in studying various stability problems. In viscous potential flow, viscous term in the Navier-Stokes equation is identically zero when the vorticity is zero but the viscous stresses are not zero. Tangential stresses are not considered in the viscous potential flow theory, and viscosity enters through normal stress balance. In this theory, no-slip condition at the boundary is not enforced so that two-dimensional solutions satisfy three-dimensional solutions. Joseph et al. [17] studied viscous potential flow of Rayleigh-Taylor instability. Funada and Joseph [18] have done the viscous potential flow analysis of Kelvin-Helmholtz instability in a channel and found that the stability criterion for viscous potential flow is given by the critical value of the relative velocity. From the above study Funada and Joseph [18] concluded that the critical value is maximum when viscosity ratio equals to the density ratio. Awasthi and Agrawal [19] have studied the viscous potential flow analysis of Kelvin-Helmholtz instability of a cylindrical interface and found that the viscosity of the fluids stabilizes the interface. The rupture of a 3D stationary free liquid film under the competing effects of surface tension and van der Waals forces has been studied by Ardekani and Joseph [20] as a linearized stability problem in a purely irrotational analysis utilizing the dissipation method.

In the present work, the viscous potential flow theory has been used to study the instability of thin liquid sheet moving in the same direction as an air stream and in the presence of uniform horizontal electric field. Both the liquid and air treats as incompressible and viscous with different kinematic viscosities and having horizontal velocities. In the present investigation, the sheet has been taken as two-dimensional, irrotational, and of constant thickness, considering only antisymmetric oscillations (predominant for instability as mentioned by Squire [4]) of the moving sheet. Normal mode technique has been used to solve the governing equations. A dispersion relation is derived and stability is discussed theoretically as well as numerically. Various neutral curves have been drawn to show the effect of various physical parameters such as Reynolds number, Weber number, electric field intensity, and ratio of permittivity of two fluids on the stability of the system. Finally, a comparison has been made between the results of present study and the results obtained by El-Sayed [15] for inviscid potential analysis.

\section{Problems Formulation}

Consider a two-dimensional irrotational sheet of viscous and dielectric liquid of density $\rho^{(1)}$, viscosity $\mu^{(1)}$, dielectric constant $\varepsilon^{(1)}$, surface tension $T$, and thickness $h=2 a$, moving with velocity $U_{1}$ along an air stream (on both sides of the sheet), which is of density $\rho^{(2)}$, viscosity $\mu^{(2)}$, dielectric constant $\varepsilon^{(2)}$, and moving with velocity $U_{2}$. Both fluids are assumed to be incompressible and irrotational. The origin is located at the mid-plane of the liquid sheet so in the equilibrium state, the equation of interface can be expressed as $y=a$. On applying small disturbances to the system, the equation of upper surface can be written as

$$
F(x, y, t)=y-a-\eta(x, t)=0,
$$

where $\eta$ represents the displacement of liquid sheet from its equilibrium position and $F$ represents the equation of the disturbed surface.

The unit outward normal to the interface is given by

$$
\mathbf{n}=\frac{\nabla F}{|\nabla F|}=\left\{1+\left(\frac{\partial \eta}{\partial x}\right)^{2}\right\}^{-1 / 2}\left(-\frac{\partial \eta}{\partial x} \mathbf{e}_{x}+\mathbf{e}_{y}\right),
$$

and the unit outward normal of the first order is given by

$$
\mathbf{n}=\left(-\frac{\partial \eta}{\partial x} \mathbf{e}_{x}+\mathbf{e}_{y}\right)
$$

In each fluid layer velocity is expressed as the gradient of the potential function $\phi(x, y, t)$, and the potential functions satisfy Laplace's equation, that is,

$$
\nabla^{2} \phi^{(j)}=0, \quad j=1,2
$$

where $\nabla^{2}=\partial^{2} / \partial x^{2}+\partial^{2} / \partial y^{2}$.

In initial state, the potential function can be written as

$$
\phi_{0}^{(j)}=U_{j} x \quad(j=1,2) .
$$

The considered system is influenced by a uniform horizontal electric field $E_{0}$ in the positive $x$-direction

$$
\mathbf{E}=E_{0} \mathbf{e}_{x}
$$

We have assumed that the quasistatic approximation is valid for the problem, and therefore, the electric field can be derived from electric scalar potential function $\psi(x, y, t)$ such that

$$
\mathbf{E}_{j}=E_{0} \mathbf{e}_{x}-\nabla \psi_{j}, \quad(j=1,2) .
$$

Gauss's law requires that the electric potentials also satisfy Laplace's equation, that is,

$$
\nabla^{2} \psi_{j}=0, \quad(j=1,2) .
$$




\section{Boundary Conditions}

(i) Kinematic condition that every particle on the interface remains on the interface leads to the following boundary conditions:

$$
\begin{gathered}
\frac{\partial \eta}{\partial t}+U_{1} \frac{\partial \eta}{\partial x}=-\frac{\partial \phi^{(1)}}{\partial y} \quad \text { at } y=-a \\
\frac{\partial \eta}{\partial t}+U_{2} \frac{\partial \eta}{\partial x}=-\frac{\partial \phi^{(2)}}{\partial y} \quad \text { at } y=a
\end{gathered}
$$

(ii) The tangential component of the electric field must be continuous across the interface, that is, $\mathbf{n} \wedge \llbracket \mathbf{E} \rrbracket=$ 0 or

$$
\frac{\partial \psi^{(2)}}{\partial x}=\frac{\partial \psi^{(2)}}{\partial x}
$$

(iii) There is discontinuity in the normal current across the interface; charge accumulation within a material element is balanced by conduction from bulk fluid on either side of the surface. Hence, the boundary condition corresponding to normal component of the electric field at the interface is given by $\mathbf{n} \cdot \llbracket \varepsilon \mathbf{E} \rrbracket=0$ or

$\varepsilon^{(2)}\left(\frac{\partial \psi^{(2)}}{\partial y}+E_{0} \frac{\partial \eta}{\partial x}\right)=\varepsilon^{(1)}\left(\frac{\partial \psi^{(1)}}{\partial y}+E_{0} \frac{\partial \eta}{\partial x}\right)$.

(iv) Interfacial condition for conservation of momentum is

$$
\begin{aligned}
& p_{2}- p_{1}-2 \mu^{(2)} \frac{\partial^{2} \phi^{(2)}}{\partial y^{2}}+2 \mu^{(1)} \frac{\partial^{2} \phi^{(1)}}{\partial y^{2}} \\
&-E_{0}\left[\varepsilon^{(2)} \frac{\partial \psi^{(2)}}{\partial x}-\varepsilon^{(1)} \frac{\partial \psi^{(1)}}{\partial x}\right]=T \frac{\partial^{2} \eta}{\partial x^{2}},
\end{aligned}
$$

where $p_{j}(j=1,2)$ are the pressure for lower and upper fluids, respectively, and this pressure can be obtained using Bernoulli's equation.

Using Bernoulli's equation for the pressure in (12) and subsequently linearizing it, we get

$$
\begin{gathered}
\rho^{(2)}\left(\frac{\partial \phi^{(2)}}{\partial t}+U_{2} \frac{\partial \phi^{(2)}}{\partial x}\right)-\rho^{(1)}\left(\frac{\partial \phi^{(1)}}{\partial t}+U_{1} \frac{\partial \phi^{(1)}}{\partial x}\right) \\
-2 \mu^{(2)} \frac{\partial^{2} \phi^{(2)}}{\partial y^{2}}+2 \mu^{(1)} \frac{\partial^{2} \phi^{(1)}}{\partial y^{2}} \\
-E_{0}\left[\varepsilon^{(2)} \frac{\partial \psi^{(2)}}{\partial x}-\varepsilon^{(1)} \frac{\partial \psi^{(1)}}{\partial x}\right]=T \frac{\partial^{2} \eta}{\partial x^{2}} .
\end{gathered}
$$

\section{Normal Mode Analysis and Dispersion Relation}

Now the normal mode technique has been used to find the solution of the governing equations. Let the interface elevation be represented by

$$
\eta=C \exp [i(n x-\omega t)]+c . c,
$$

where $C$ is constant, $k$ is the real wave number, $\omega$ is the growth rate, and c.c. refers to the complex conjugate of the preceding term.

On solving (3) and (8) with the help of boundary conditions, we get

$$
\begin{gathered}
\phi^{(1)}=i\left(\frac{\omega}{n}-U_{1}\right) C \frac{\sinh n y}{\cosh n a} \exp [i(n x-\omega t)]+c . c, \\
\phi^{(2)}=-i\left(\frac{\omega}{n}-U_{2}\right) C \exp \{-n(y-a)\} \\
\quad \times \exp [i(n x-\omega t)]+c . c \\
\psi^{(1)}=\frac{i E_{0}\left(\varepsilon^{(2)}-\varepsilon^{(1)}\right)}{\left(\varepsilon^{(2)}+\varepsilon^{(1)} \operatorname{coth} n a\right)} C \frac{\sinh n y}{\sinh n a} \\
\quad \times \exp [i(n x-\omega t)]+c . c \\
\psi^{(2)}=\frac{i E_{0}\left(\varepsilon^{(2)}-\varepsilon^{(1)}\right)}{\left(\varepsilon^{(2)}+\varepsilon^{(1)} \operatorname{coth} n a\right)} C \exp \{-n(y-a)\} \\
\quad \times \exp [i(n x-\omega t)]+c . c .
\end{gathered}
$$

Substituting the values of $\eta, \phi^{(1)}, \phi^{(2)}, \psi^{(1)}$, and $\psi^{(2)}$ in (13), we get the dispersion relation

$$
\begin{aligned}
& \rho^{(1)}\left(\frac{\omega}{n}-U_{1}\right)^{2} \tanh n a+\rho^{(2)}\left(\frac{\omega}{n}-U_{2}\right)^{2} \\
& -2 i \mu^{(1)}\left(\frac{\omega}{n}-U_{1}\right) n \tanh n a-2 i \mu^{(2)} \\
& \quad \times\left(\frac{\omega}{n}-U_{2}\right) n-\frac{E_{0}^{2}\left(\varepsilon^{(2)}-\varepsilon^{(1)}\right)^{2}}{\varepsilon^{(2)}+\varepsilon^{(1)} \operatorname{coth} n a}=T n .
\end{aligned}
$$

Dividing (16) by $\rho^{(1)} U_{1}^{2}$ and calling $\rho^{(2)} / \rho^{(1)}=\rho, \mu^{(2)} / \mu^{(1)}=$ $\mu, \varepsilon^{(2)} / \varepsilon^{(1)}=\varepsilon, \sigma=\omega / n U_{1}, k=n a$, and $E^{2}=E_{0}^{2} \varepsilon^{(1)} / \rho^{(1)} U_{1}^{2}$, we can get

$$
\begin{aligned}
(\sigma-1)^{2} \tanh k+\rho(\sigma-U)^{2} \\
-i \frac{2 k}{\operatorname{Re}} \times[(\sigma-1) \tanh k+\mu(\sigma-U)] \\
=\left[\frac{E^{2}(\varepsilon-1)^{2}}{\varepsilon+\operatorname{coth} k}+W k\right],
\end{aligned}
$$

where $W=T / \rho^{(1)} U_{1}^{2} a$ represents the Weber number and $\operatorname{Re}=\rho^{(1)} U_{1} a / \mu^{(1)}$ denotes Reynolds number.

Equation (17) can be written as

$$
D(\sigma, k)=a_{0} \sigma^{2}+\left(a_{1}+i b_{1}\right) \sigma+\left(a_{2}+i b_{2}\right)=0,
$$

where $a_{0}=\tanh k+\rho, a_{1}=-2(\tanh k+\rho U), b_{1}=$ $-(2 k / \operatorname{Re})(\tanh k+\mu), a_{2}=\left(\tanh k+\rho U^{2}\right)-E^{2}(\varepsilon-1)^{2} /(\varepsilon+$ $\operatorname{coth} k)-W k$, and $b_{2}=(2 k / \operatorname{Re})(\tanh k+\mu U)$. 
Let $\sigma=\sigma_{R}+i \sigma_{\mathrm{I}}$, separating the real and imaginary parts of (18), we have

$$
\begin{gathered}
a_{0}\left(\sigma_{R}^{2}-\sigma_{I}^{2}\right)+\left(a_{1} \sigma_{R}-b_{1} \sigma_{I}\right)+a_{2}=0, \\
\sigma_{R}=-\frac{a_{1} \sigma_{I}+b_{2}}{2 a_{0} \sigma_{I}+b_{1}} .
\end{gathered}
$$

Eliminating the value of $\sigma_{R}$ from the previous equations, we get a quartic equation in $\sigma_{\mathrm{I}}$ as

$$
A_{4} \sigma_{I}^{4}+A_{3} \sigma_{I}^{3}+A_{2} \sigma_{I}^{2}+A_{1} \sigma_{I}+A_{0}=0,
$$

where $A_{4}=-4 a_{0}^{3}, A_{3}=-8 a_{0}^{2} b_{1}, A_{2}=4 a_{0}^{2} a_{2}-5 a_{0} b_{1}^{2}-a_{0} a_{1}^{2}$, $A_{1}=4 a_{0} a_{2} b_{1}-b_{1}^{3}-a_{1}^{2} b_{1}$, and $A_{0}=a_{0} b_{2}^{2}-a_{1} b_{1} b_{2}+a_{2} b_{1}^{2}$.

From (20), we can get the value of maximum growth rate $\sigma_{I m}$ and corresponding wave number $k_{m}$. The neutral curves can be obtained from (20) by putting $\sigma_{I}=0$, so (20) reduces to

$$
A_{0}=0, \quad \text { that is, } a_{0} b_{2}^{2}-a_{0} b_{1} b_{2}-a_{2} b_{1}^{2}=0 .
$$

Putting the values of $a_{0}, a_{1}, a_{2}, b_{1}$, and $b_{2}$ in the above equation, we get

$$
\begin{aligned}
(1-U)^{2} & \left\{\mu^{2} \tanh k+\rho \tanh ^{2} k\right\} \\
& =\left[\frac{E^{2}(\varepsilon-1)^{2}}{\varepsilon+\operatorname{coth} k}+W k\right]\{\tanh k+\mu\}^{2} .
\end{aligned}
$$

In practical applications, $k$ is very small [4], then taking the approximation $\tanh k \approx k$ in (22), we have

$$
(1-U)^{2}\left\{\mu^{2}+\rho k\right\}=\left[\frac{E^{2}(\varepsilon-1)^{2}}{\varepsilon k+1}+W\right]\{k+\mu\}^{2} .
$$

Hence, for instability it must be

$$
\frac{(1-U)^{2}}{W}>\left[\frac{E^{2}(\varepsilon-1)^{2}}{W(\varepsilon k+1)}+1\right] \frac{\{k+\mu\}^{2}}{\left\{\mu^{2}+\rho k\right\}} .
$$

From the expression (24) the following conclusions can be easily drawn.

(1) If fluids are inviscid, that is, $\mu=0$, it is reduced to dispersion relation as obtained by El-Sayed [15].

(2) In the absence of electric field and viscosity, (24) will represent the same dispersion relation as obtained by Rashed et al. [9].

(3) In the limit when $E, U$, and $\mu$ tend to zero, (24) is reduced to dispersion relation as obtained by Squire [4].

The condition of instability of the liquid sheet can also be written as

$$
W<\left[(1-U)^{2} \frac{\left\{\mu^{2}+\rho k\right\}}{\{k+\mu\}^{2}}-\frac{E^{2}(\varepsilon-1)^{2}}{(\varepsilon k+1)}\right] .
$$

Hence, the instability occurs if Weber number is less than the critical value of Weber number $W_{c}$; otherwise system is stable where $W_{c}$ is given by

$$
W_{c}=\left[(1-U)^{2} \frac{\left\{\mu^{2}+\rho k\right\}}{\{k+\mu\}^{2}}-\frac{E^{2}(\varepsilon-1)^{2}}{(\varepsilon k+1)}\right] .
$$

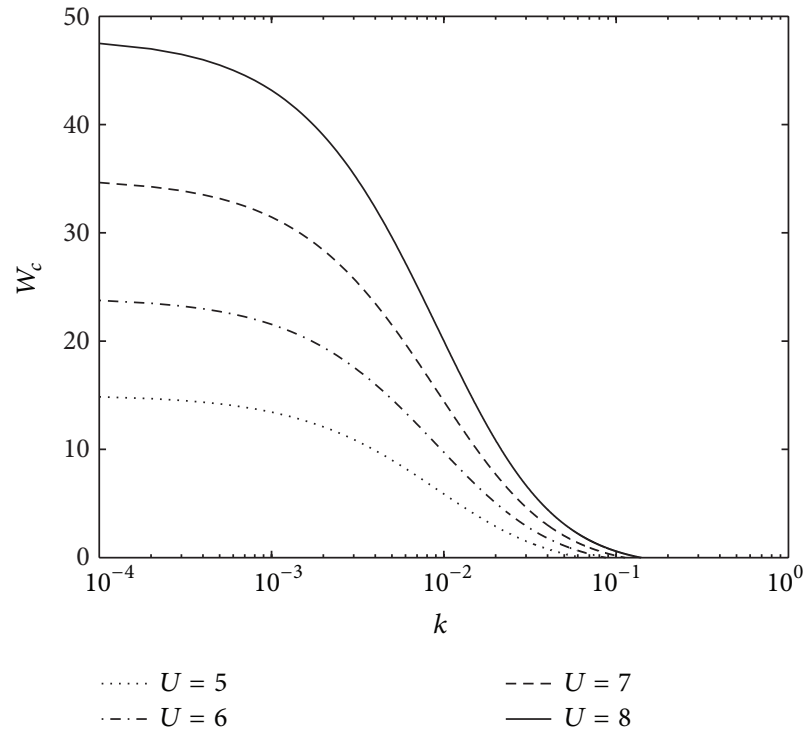

FIgURE 1: The neutral curves of Weber number for the different values of velocity ratio when $E=1, \mu=0.018, \rho=0.0012$.

\section{Results and Discussion}

In this section the numerical computation has been carried out using the expressions (26) and (23) presented in the previous section. Water and air have been taken as working fluids identified with phase 1 and phase 2, respectively. Following parametric values have been taken.

$\rho^{(1)}=1.0 \mathrm{gm} / \mathrm{cm}^{3}, \rho^{(2)}=0.001 \mathrm{gm} / \mathrm{cm}^{3}, \mu^{(1)}=$ 0.01 poise, $\mu^{(2)}=0.00018$ poise, $\varepsilon^{(1)}=80.37 \mathrm{~F} / \mathrm{cm}, \varepsilon^{(2)}=$ $1.0 \mathrm{~F} / \mathrm{cm}$, and $T=72.3$ dyne $/ \mathrm{cm}$.

The neutral curves of Weber number for the different values of velocity ratio of two fluids have been drawn in Figure 1. The region above the curves denotes the stable region, while below region is the region of instability. As velocity ratio increases, stable region decreases, and therefore, the velocity ratio of two fluids has destabilizing effect. In other words, one can say that the air velocity has destabilizing effect while liquid velocity plays stabilizing role.

The effect of Weber number on the neutral curves of velocity ratio of two fluids has been shown in Figure 2 . Neutral curves for velocity ratio divide the plane into the stable region (below the curve) and unstable region (above the curve). As Weber number increases, the region of stability also increases for the same value of other parameters. The Weber number is directly proportional to the surface tension of the liquid. Therefore, as surface tension increases, the disintegration of the liquid sheet decreases. Therefore, it is concluded that the effect of surface tension resists the occurrence and development of instability.

In Figure 3, the effect of ratio of viscosity of two fluids has been studied. As viscosity ratio increases, the stable region decreases, and this shows that viscosity ratio of two fluids has destabilizing effect on the aerodynamic instability of the liquid sheet. The viscosity ratio is directly proportional to the air viscosity and inversely proportional to the liquid 


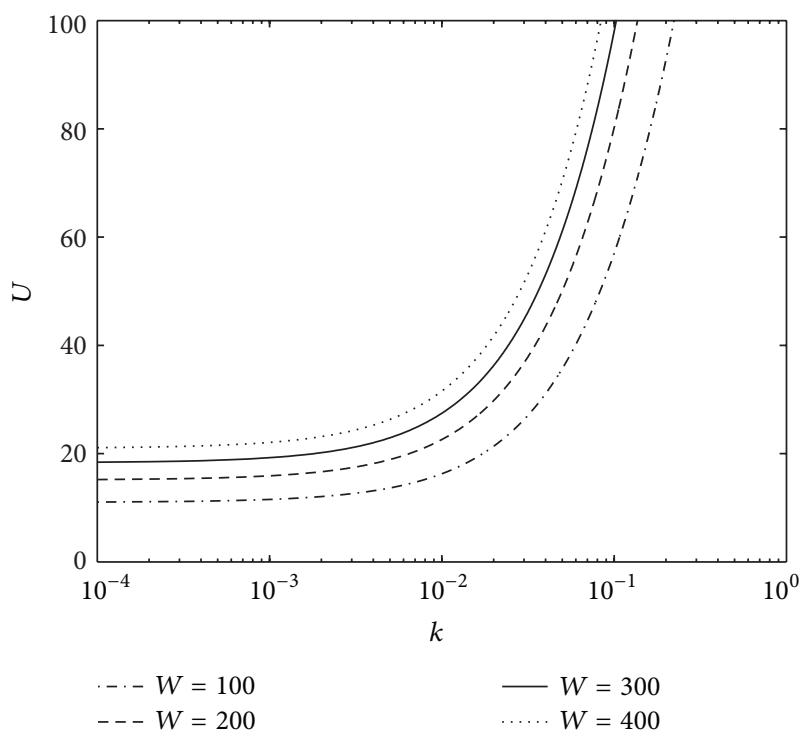

FIGURE 2: The neutral curves of velocity ratio for the different values of Weber number when $E=0, \mu=0.018, \rho=0.0012$.

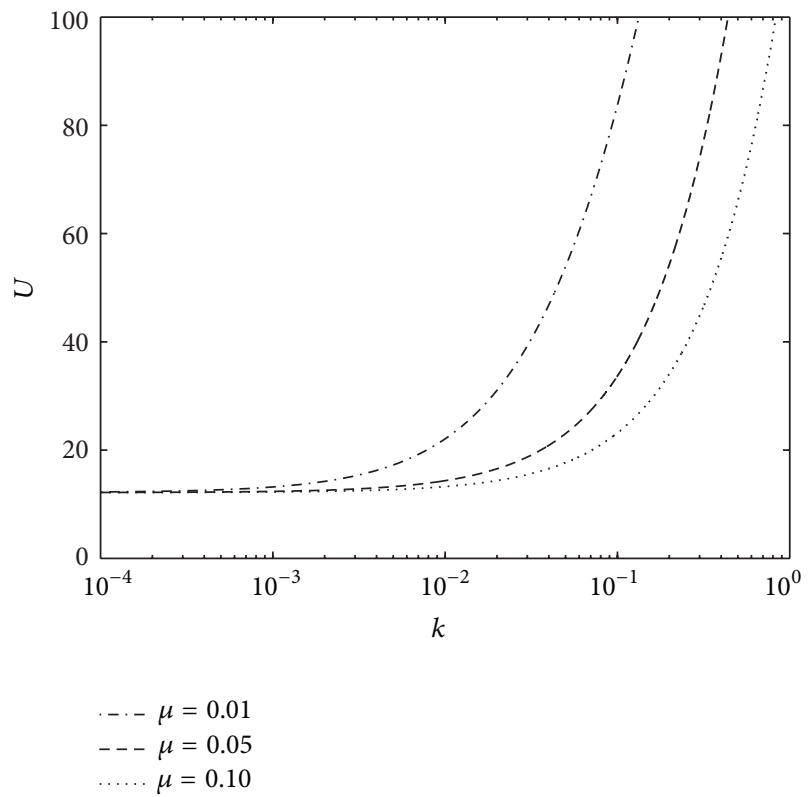

FIGURE 3: The neutral curves of velocity ratio for the different values of viscosity ratio of two fluids when $E=5, W=100, \rho=0.0012$.

viscosity. Hence, air viscosity has destabilizing effect on the aerodynamic stability analysis of a liquid sheet, while liquid viscosity plays a stabilizing role. As the air viscosity increases, the air flow is impeded and instability occurs.

The effect of electric field intensity $E$ on the neutral curves of velocity ratio has been shown in Figure 4 when Weber number $W=100$ and viscosity ratio $\mu=0.018$. It has been noticed that the increase in electric field intensity enlarges the stable region. Hence electric field has stabilizing effect on the instability of the liquid sheet. If electric field is

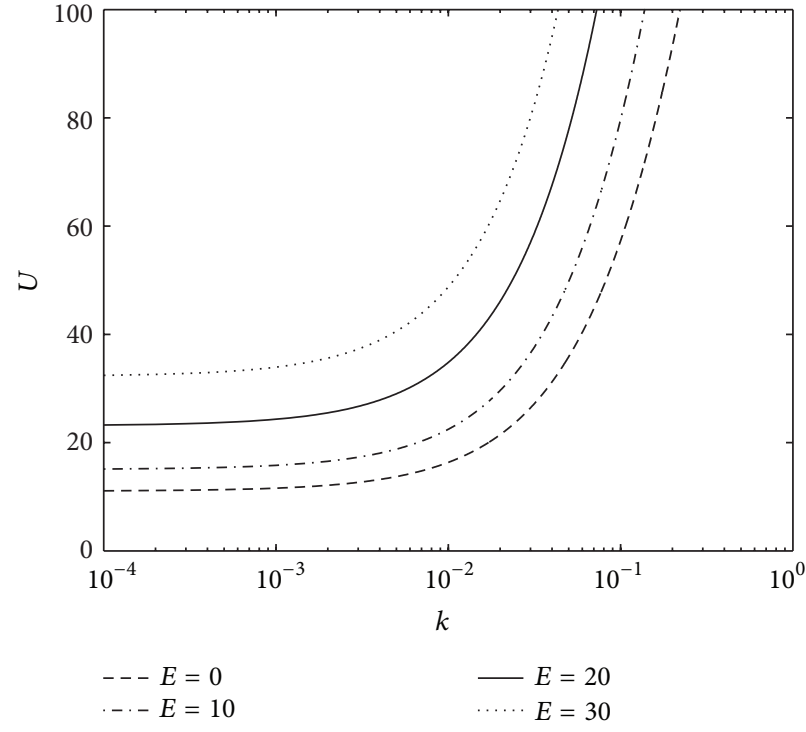

FIgURE 4: The neutral curves of velocity ratio for the different values of electric field intensity when $W=100, \mu=0.018, \rho=0.0012$.

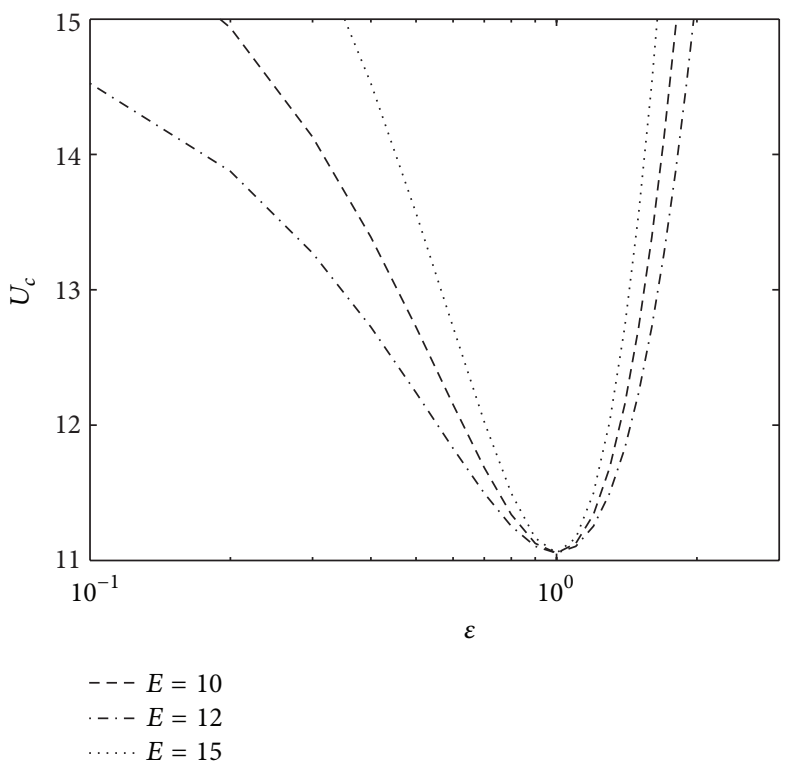

FIgURE 5: The neutral curves of velocity ratio versus ratio of dielectric constants of two fluids for the different values of electric field intensity when $W=100, \mu=0.018, \rho=0.0012$.

present in the analysis, the term contributed from the applied electric field is added in the right hand side of (23) and so that critical value of velocity ratio increases. This shows that electric field has stabilizing influence.

In Figure 5, the variation of critical value of velocity ratio $U$ with the permittivity ratio of two fluids $\varepsilon$ for different values of electric field intensity $E=10,12$, and 15 has been shown for Weber number $W=100$. The figure shows that as the ratio of the permittivities of the two fluids increases, the critical value of relative velocity first decreases and then increases. It shows that $\varepsilon$ plays dual role, 
that is, destabilizing as well as stabilizing effect in the stability analysis.

\section{Conclusions}

The instability of a thin sheet of viscous and dielectric liquid moving in the same direction as an air stream in the presence of a uniform horizontal electric field has been investigated using viscous potential flow theory. Both fluids are taken as incompressible and viscous with different kinematic viscosities. A dispersion relation has been derived, and stability is discussed theoretically as well as numerically. Stability criterion is given in the terms of velocity ratio of two fluids as well as critical value of applied electric field. The system is unstable when the Weber number is less than the critical value of Weber number; otherwise, it is stable. It has been found that aerodynamic-enhanced instability occurs if the velocity ratio is greater than a critical value related to the ratio of the air and liquid viscosities, Weber number, the electric field, and the dielectric constant values. The electric field is found to have a stabilizing effect while dielectric constant plays dual role in the stability criterion. Surface tension has stability effect on the aerodynamic instability of a liquid sheet. Liquid viscosity has stabilizing effect in the stability analysis, while air viscosity has destabilizing effect.

\section{References}

[1] A. H. Lefebvre, Atomization and Sprays, Hemisphere, New York, NY, USA, 1989.

[2] K. Masters, Spray Drying Handbook, Wiley, New York, NY, USA, 4th edition, 1985.

[3] S. P. Lin and W. C. Liu, "Instability of film coating of wires and tubes," AIChE Journal, vol. 21, no. 4, pp. 775-782, 1975.

[4] H. B. Squire, "Investigation of the instability of a moving liquid film," British Journal of Applied Physics, vol. 4, no. 6, article no. 302, pp. 167-169, 1953.

[5] W. W. Hagerty and J. F. Shea, "A study of the stability of plane fluid sheets," Journal of Applied Mechanics, vol. 22, p. 509, 1955.

[6] R. P. Fraser, "The fluid kinetics of applications of pesticidal chemicals," Advances in Pest Control Research, vol. 11, pp. 1-106, 1958.

[7] N. Dombrowski and W. R. Johns, "The aerodynamic instability and disintegration of viscous liquid sheets," Chemical Engineering Science, vol. 18, no. 3, pp. 203-214, 1963.

[8] J. G. H. Joosten, A. Vrij, and H. M. Fijnaut, in Physicochemical Hydrodynamics, Proceedings ofthe International Conference on Physical Chemistry and Hydrodynamics, D. B. Spalding, Ed., vol. II, p. 639, Advance Publications, Guernsey, UK, 1978.

[9] M. I. I. Rashed, M. A. Ghazi, and H. Elbanna, "Aerodynamic instability of a liquid sheet sprayed with an air stream," Journal of Physics D, vol. 12, no. 10, article 009, pp. 1679-1684, 1979.

[10] E. A. Ibrahim and S. L. Jackson, "Spatial instability of a liquid sheet in a compressible gas," Journal of Colloid and Interface Science, vol. 180, no. 2, pp. 629-631, 1996.

[11] E. A. Ibrahim and E. T. Akpan, "Liquid sheet instability," Acta Mechanica, vol. 131, no. 3-4, pp. 153-167, 1998.

[12] E. A. Ibrahim, "Instability of a liquid sheet of parabolic velocity profile," Physics of Fluids, vol. 10, no. 4, pp. 1034-1036, 1998.
[13] A. A. Ibrahim and M. A. Jog, "Nonlinear instability of an annular liquid sheet exposed to gas flow," International Journal of Multiphase Flow, vol. 34, no. 7, pp. 647-664, 2008.

[14] J. R. Melcher, Continuum Electromechanics, MIT Press, Cambridge, Mass, USA, 1981.

[15] M. F. El-Sayed, "Electro-aerodynamic instability of a thin dielectric liquid sheet sprayed with an air stream," Physical Review E, vol. 60, no. 6B, pp. 7588-7591, 1999.

[16] D. D. Joseph and T. Y. Liao, "Potential flows of viscous and viscoelastic fluids," Journal of Fluid Mechanics, vol. 265, pp. 123, 1994.

[17] D. D. Joseph, J. Belanger, and G. S. Beavers, "Breakup of a liquid drop suddenly exposed to a high-speed airstream," International Journal of Multiphase Flow, vol. 25, no. 6-7, pp. 1263-1303, 1999.

[18] T. Funada and D. D. Joseph, "Viscous potential flow analysis of Kelvin-Helmholtz instability in a channel," Journal of Fluid Mechanics, vol. 445, pp. 261-283, 2001.

[19] M. K. Awasthi and G. S. Agrawal, "Viscous Potential flow analysis of Kelvin-Helmholtz instability of cylindrical interface," International Journal of Applied Mathematics and Computation, vol. 3, no. 2, pp. 131-138, 2011.

[20] A. M. Ardekani and D. D. Joseph, "Instability of stationary liquid sheets," Proceedings of the National Academy of Sciences of the United States of America, vol. 106, no. 13, pp. 4992-4996, 2009. 

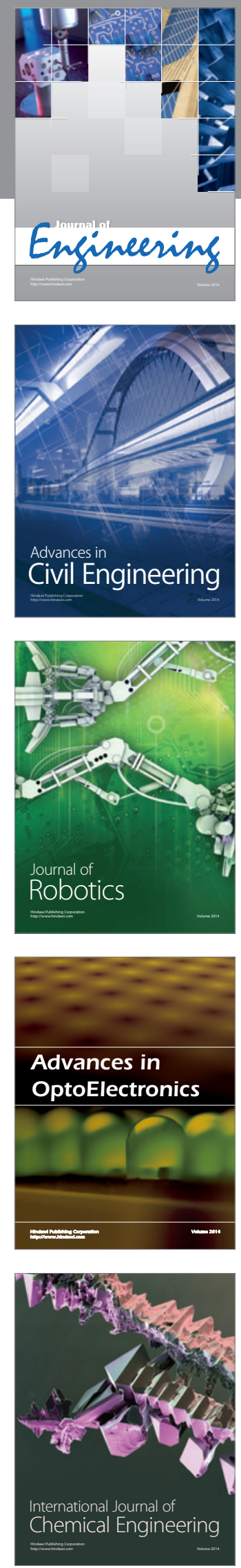

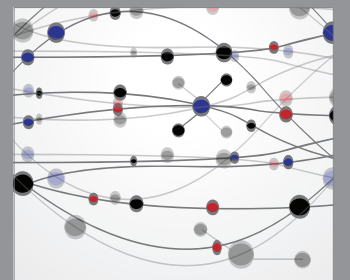

The Scientific World Journal
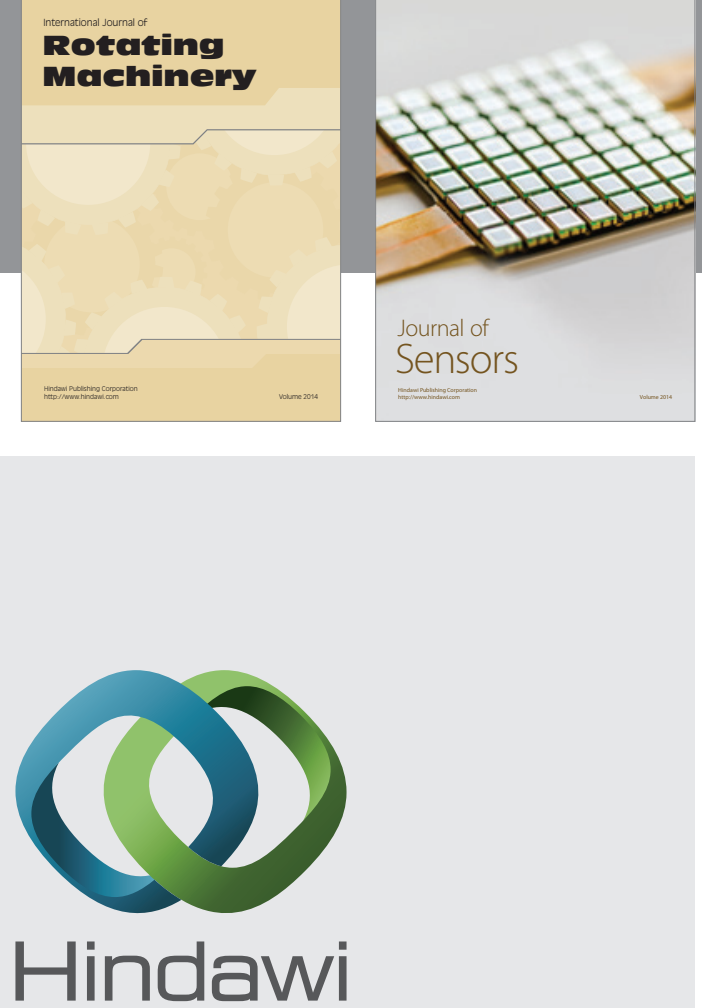

Submit your manuscripts at http://www.hindawi.com
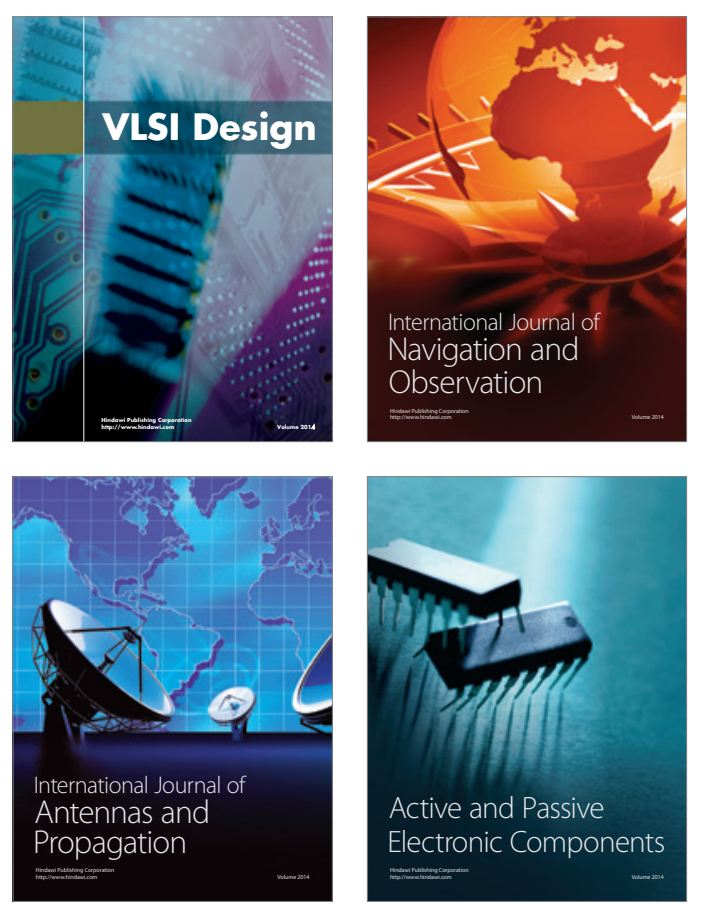
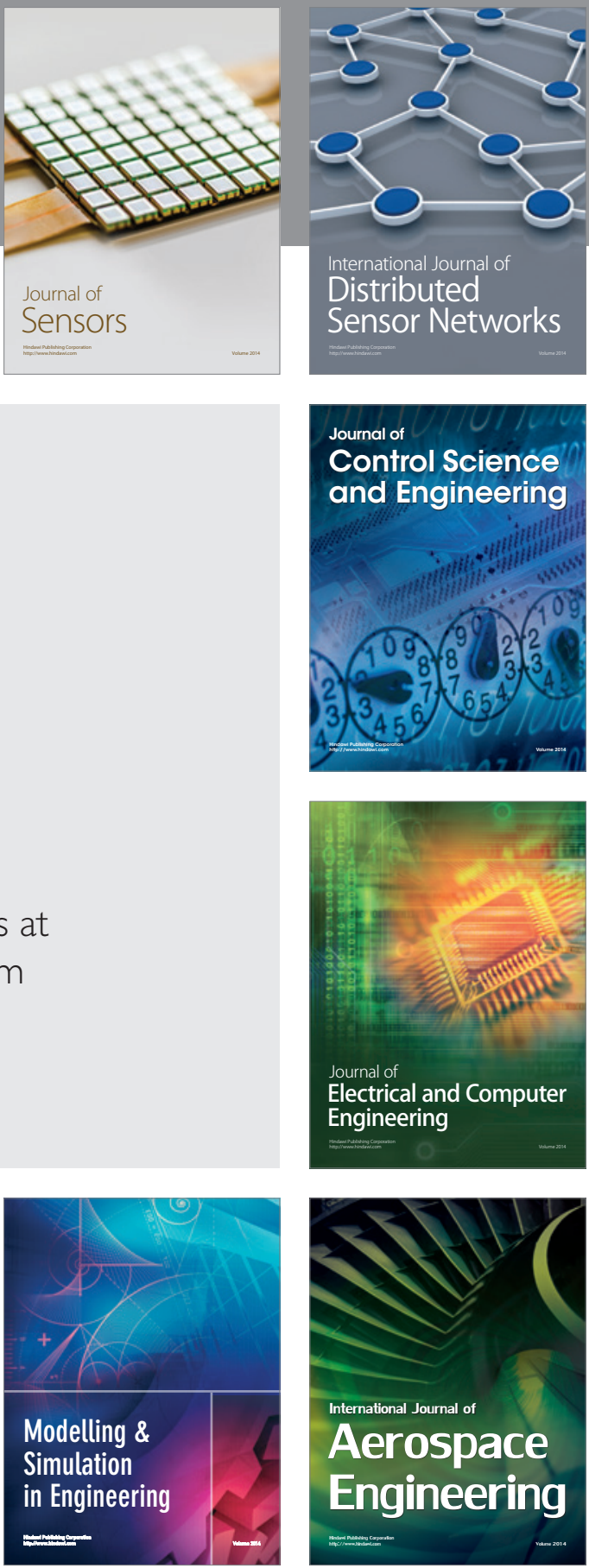

Journal of

Control Science

and Engineering
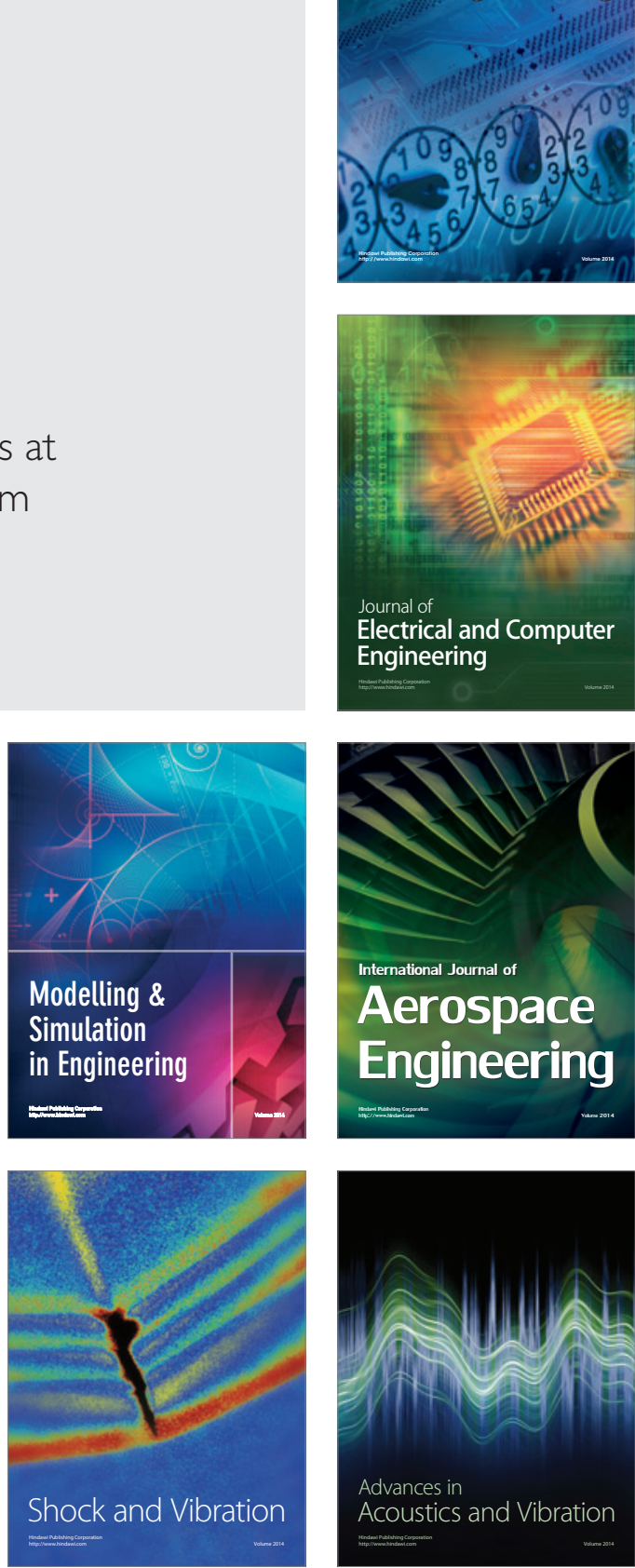